S3 Table. Primer sets for real-time PCR

\title{
Rat Acta2
}

5'-AGGATGCAGAAGGAGATCACAG-3'

5'-CTGGAAGGTAGATAGAGAAGCC-3'

\section{Rat $C d h 1$}

5'-GGAGAAGAAGACCAGGACTTTG-3'

5'-GATGAAGTTCCCGATTTCATCAG-3'

\section{Rat $A c t b 1$}

5'-TATGCCAACACAGTGCTGTCTG-3'

5'-CTCAGGAGGAGCAATGATCTTG-3' 